\title{
Transtorno de pânico e hipocondria
}

Panic disorder and hypocondriasis

\author{
Monica Dib, Alexandre M. Valença e Antonio Egidio Nardi
}

\section{Resumo}

\begin{abstract}
A hipocondria é associada a diversos transtornos de ansiedade, sobretudo ao transtorno de pânico (TP). Estima-se que 50\% a 70\% dos pacientes com TP tenham sintomas hipocondríacos e que $13 \%$ a $17 \%$ dos hipocondríacos tenham TP associado. Considera-se que há co-morbidade com hipocondria no TP quando as preocupações com saúde não se restringem aos sintomas das crises de pânico. Relatamos um caso de uma paciente que, durante seu acompanhamento, evoluiu com hipocondria e transtorno de pânico associados. Discutimos as manifestações psiquiátricas manifestadas pela paciente, assim como analisamos aspectos conceituais, diagnósticos e prognósticos. Palavras-chave: transtorno de pânico, hipocondria, diagnóstico diferencial, co-morbidade.
\end{abstract}

\begin{abstract}
Hypocondriasis is associated with several anxiety disorders, including panic disorder. The available estimates of panic disorder patients with identified hypochondriacal symptoms are $50 \%$ to $70 \%$. Complimentary, $13 \%$ to $17 \%$ of hypochondriac patients were associated with panic disorder. Comorbidity and hypocondriasis occur when health care issues are not delimited by panic disorder symptoms. We reported a patient that, during the follow-up period, has evolved to an associated hypocondriasis and panic disorder scenario. The psychiatric symptoms were properly addressed and discussed, as well the associated conceptual aspects, diagnoses and prognosis.
\end{abstract}

Key words: panic disorder, hypochondriasis, diagnosis differential, comorbidity. 


\section{Introdução}

O transtorno do pânico (TP) foi conhecido por diferentes terminologias, mas sempre com certa precisão descritiva, desde a Antigüidade. Foi caracterizado como entidade nosológica autônoma oficial apenas a partir da terceira revisão do Manual Diagnóstico e Estatístico de Transtornos Mentais (DSM-III), com base nos trabalhos de Donald Klein nos anos 1960. Anteriormente o TP integrava oficialmente o grupo das neuroses de ansiedade e das neuroses fóbicas. Palpitações, taquicardia, sudorese, tremores, sensação de falta de ar, asfixia, desconforto torácico, náusea, desconforto abdominal, medo de morrer, parestesias e calafrios podem levar o paciente com TP a ter preocupações hipocondríacas.

A hipocondria é a preocupação com medo ou a idéia de sofrer de uma enfermidade com base numa interpretação errônea de sintomas ou funções corporais, em que o indivíduo teme sofrer de uma doença grave (critério A do DSM-IV) apesar de avaliações e garantias médicas apropriadas (critério $B$ do DSM-IV). O transtorno hipocondríaco na Classificação Internacional de Doenças (CID-10) é similar àquele no DSM-IV, incluindo queixas somáticas persistentes ou preocupação duradoura com a aparência física. Mesmo com avaliação e garantias médicas apropriadas, os hipocondríacos contam com preocupações não-delirantes de doença grave baseadas na interpretação equivocada de manifestações somáticas, o que traz prejuízos com duração mínima de seis meses (DSM-IV).

A discussão dos critérios conceituais e diagnósticos da hipocondria é freqüente (Abramowitz et al., 2002; Noyes et al., 2003; Ornbol et al., 2004), assim como a sua relação com o TP (Torres et al., 2002; Hiller et al., 2005; Scrignar, 2004). A hipocondria é observada em $4 \%$ a $6 \%$ dos indivíduos na área médica (Barsky, 2001). Dois terços dos pacientes com hipocondria apresentam outro transtorno psiquiátrico associado, incluindo transtorno depressivo maior (40\% dos casos); TP (10\% a $20 \%)$, transtorno obsessivo compulsivo (TOC) $(5 \%$ a $10 \%)$ e transtorno de ansiedade generalizada (Torres et al., 2002).

\section{Relato de caso}

F., 29 anos, branca, casada, católica, balconista, residente no Rio de Janeiro.

Queixa principal (QP): arritmia e falta de ar.

Em 2002, a paciente apresentou um quadro que denominou de crise nervosa grave, apresentando dispnéia, taquicardia e sudorese de extremidades, tendo sido levada para a unidade de emergência de um hospital próximo pelos familiares. Realizou exame físico, exames laboratoriais e eletrocardiograma, todos normais. A suspeita inicial de infarto agudo do miocárdio (IAM) foi descartada.

A partir desse acontecimento $F$. passou a freqüentar diversos serviços de saúde na busca de um diagnóstico para o que denominava ser um problema cardíaco, argumentando que seu pai e seu avô paterno morreram de IAM. Desde então F. peregrinou por diversos serviços de cardiologia com o intuito de realizar um check-up cardiológico, sendo internada com suspeita de IAM toda vez que sentia os seguintes sintomas paroxísticos: sudorese, taquicardia, dispnéia, tremor e sudorese de extremidades e aperto no peito.

Em meados de 2004 F. novamente se submeteu a exames cardiológicos, queixando-se dos mesmos sintomas. Os exames nada constataram de anormal. Um cardiologista encaminhou-a ao atendimento psiquiátrico. Compareceu ao Instituto de Psiquiatria da Universidade Federal do Rio de Janeiro (IPUB/UFRJ) em novembro de 2004. Veio acompanhada do marido, pois temia andar na rua sozinha e passar mal. Fazia uso de $5 \mathrm{mg}$ diários de diazepam, prescrição feita pelo médico que a encaminhou ao IPUB. Durante a entrevista aventou diversas vezes a hipótese de sofrer de arritmia cardíaca. Prescrição inicial: paroxetina, $10 \mathrm{mg} / \mathrm{dia}$, no período noturno e manutenção do diazepam $10 \mathrm{mg} / \mathrm{dia}$.

A sintomatologia se reduziu em intensidade e freqüência no primeiro ano de tratamento.

Durante o período entre as consultas, F. entrou em contato diversas vezes para discutir os efeitos colaterais da paroxetina que leu na bula. Indagou sobre o uso crônico do antidepressivo e se este poderia promover aumento súbito da pressão arterial ou piorar sua arritmia. Parou o tratamento de forma abrupta duas vezes, temendo que as medicações pudessem surtir efeitos colaterais que prejudicassem seu aparelho cardiovascular. Em uma de suas consultas, no final de novembro de 2005, sugerimos um tratamento psicoterápico. F. reagiu de forma hostil, dizendo: "não sou problemática", e referindo que seus familiares sempre reclamam de que ela tem mania de doença.

Atualmente F. faz uso de $40 \mathrm{mg} /$ dia de paroxetina e $10 \mathrm{mg} /$ dia de diazepam. A freqüência e a intensidade dos sinais e sintomas do TP diminuíram significativamente. Ela já consegue ir para seu trabalho sozinha e refere que o tratamento psiquiátrico ajuda a tratar seu nervosismo. No entanto F. ainda não descarta a hipótese de um problema cardíaco ainda não-diagnosticado.

\section{Discussão}

A hipocondria está incluída nos transtornos somatoformes (DSM-IV), caracterizados pela presença de sintomas físicos que sugerem uma condição médica geral, mas que não são explicados por uma condição clínica, por efeito de substâncias ou por um outro transtorno mental, distinguindo-se dos demais transtornos somatoformes quando a idéia de ter uma doença grave ocorrer não somente no curso do transtorno de somatização (DSM-IV). O diagnóstico de hipocondria vem sendo discutido por diversos autores em estudos clínicos e pesquisas sistemáticas que utilizam escalas apropriadas (Hiller et al., 2005; Noyes et al., 2003; Longley, 2005).

Um estudo de prevalência em três grandes capitais brasileiras mostra que o transtorno somatoforme acomete mais mulheres do que homens: 5,2: 1 (Brasília); 4,3: 1 (São Paulo) e 2,3: 1 (Porto Alegre) entre mulheres e homens, respectivamente (Naomar et al., 1997). Num estudo realizado no Hospital Universitário Clementino Fraga Filho (HUCFF), da UFRJ, a taxa de prevalência de hipocondria foi $6,15 \%$ (Brasil MA, 1997). 
A distinção entre transtornos de ansiedade e somatização pode ser muito difícil. No TP podem ocorrer sintomas somáticos múltiplos, mas isto deve ocorrer durante 0 ataque de pânico. O TP pode coexistir com transtornos somatoformes quando os sintomas somáticos ocorrerem fora dos ataques de pânico, situação em que ambos os diagnósticos podem ser feitos (Hiller et al., 2005).

A hipocondria e o TP envolvem uma preocupação excessiva com doenças físicas, atenção seletiva corporal, interpretações catastróficas de sinais ou sensações corporais como alguma doença grave e comportamentos repetidos de verificação da saúde, como pode ser visto no caso relatado. A co-morbidade entre TP e hipocondria parece ser maior que o esperado ao acaso, e, ao contrário do TP, onde as preocupações sobre a sintomatologia física tendem a desaparecer com o controle das crises, no curso da hipocondria essas preocupações são geralmente de caráter crônico e intermitente (Shinoda et al., 1999; Katerrndahl, 1999; Torres et al., 2002). No caso da paciente, suas preocupações com seu estado de saúde parecem extrapolar o TP, apesar do controle da sintomatologia da doença, necessitando de uma abordagem específica.

\section{Conclusão}

Um manejo diferencial desses pacientes é necessário, pois a relação médico/paciente fica comprometida pela falta de confiança do paciente em função do prejuízo da crítica, pela dificuldade de delegar o controle ao médico, pela vigilância desses pacientes nas manifestações somáticas e nos efeitos colaterais dos medicamentos e também pela inabilidade do médico em lidar com pessoas com sinais e sintomas somáticos funcionais diversos (Shinoda et al., 1999; Katerrndahl, 1999; Mayou, Farmer, 2002; De Waal, 2004). São fundamentais a detecção e a abordagem adequada desses casos difíceis para que se elabore um vínculo com o paciente e a estratégia terapêutica adequada a fim de evitar o uso excessivo e iatrogênico dos serviços de saúde (Noyes et al., 2003; Bass e May, 2005), evitando assim o retardo no diagnóstico e no tratamento adequado.

\section{Referências}

Abramowitz JS A, Schwartz SA, Whiteside SP. A contemporary conceptual model of hypochondriasis. Mayo Clin Proc, 77: 1323-30, 2002.

American Psychiatric Association (APA). Diagnostic and Statistical Manual of Mental Disorders. Terceira Revisão. DSM-III; 1980.

American Psychiatric Association (APA). Diagnostic and Statistical Manual of Mental Disorders. Quarta Revisão. DSM-IV; 1994.

Bass C, May S. Chronicle multiple functional somatic symptoms. BMJ, 325(3): 265-8, 2002.

Barsky AJ. The patient with hypochondriasis. N Engl J Med, 345: 1395-99, 2001.

Brasil MA. A unidade psiquiátrica em hospital geral. 1997. Tese de doutorado. IPUB/UFRJ, Rio de Janeiro.

De Waal MWM, Arnold IA, Eekof JAH. Somatoform disorders in general practice: prevalence, functional impairment and comorbidity with anxiety and depressive disorders. Br J Psychiatry, 184: 470-6,2004.

Fink P, Ornbol E, Sparle KC, Frostholm L, Olesen F. A new empirically established hypochondriasis diagnosis. Am J Psychiatry, 161: 168091, 2004.

Hiller W, Leibbrand R, Rief W, Fichter MM. Differentiating hypochondriasis from panic disorder. J Ansiety Dis, (19): 29-49, 2005.
Katerndahl DA. IIIness attitudes and coping process in subjects with panic attacks. J Nerv Ment Dis, 187: 562-6, 1999.

Mayou M, Farmer A. Functional somatic symptoms and syndromes. BMJ, 325(3): 265-8, 2002.

Noyes R, et al. Test of an interpersonal model of hypochondriasis. Psychosom Med, 65: 292-300, 2003.

Noyes R, Longley S. Assessment of the hypochondriasis domain: the multidimensional inventory of hypochondriacal traits. Psychol Assess, 17(1): 3-14, 2005.

Organização Mundial da Saúde (OMS). Classificação de transtornos mentais e de comportamento da CID-10. Porto Alegre: Artes Médicas; 1993.

Scignar CB. Hypocondriasis or panic disorder? J La State Med Soc 156(4): 200-2, 2004.

Shinoda N, Kodama K, Sakamoto T, Yamanouchi N, Takahashi T, et al. Predictors of 1-year outcome for patients with panic disorder. Compr Psychiatry, 40: 39-43, 1999.

Torres AR, Crepaldi AL. Sobre o transtorno de pânico e a hipocondria: uma revisão. Rev Bras Psiquiatr, 24(3): 144-51, 2002. 